\title{
Detection of Requirement Errors and Faults via a Human Error Taxonomy: A Feasibility Study
}

\author{
Wenhua Hu \\ The University of Alabama \\ whu10@crimson.ua.edu
}

\author{
Jeffrey C. Carver \\ The University of Alabama \\ carver@cs.ua.edu
}

\author{
Vaibhav K. Anu \\ North Dakota State University \\ vaibhav.anu@ndsu.edu
}

\author{
Gursimran S. Walia \\ North Dakota State University \\ gursimran.walia@ndsu.edu
}

\author{
Gary Bradshaw \\ Mississippi State University \\ glb2@msstate.edu
}

\begin{abstract}
Background: Developing correct software requirements is important for overall software quality. Most existing quality improvement approaches focus on detection and removal of faults (i.e. problems recorded in a document) as opposed identifying the underlying errors that produced those faults. Accordingly, developers are likely to make the same errors in the future and fail to recognize other existing faults with the same origins. Therefore, we have created a Human Error Taxonomy (HET) to help software engineers improve their software requirement specification (SRS) documents. Aims: The goal of this paper is to analyze whether the HET is useful for classifying errors and for guiding developers to find additional faults. Methods: We conducted a empirical study in a classroom setting to evaluate the usefulness and feasibility of the HET. Results: First, software developers were able to employ error categories in the HET to identify and classify the underlying sources of faults identified during the inspection of SRS documents. Second, developers were able to use that information to detect additional faults that had gone unnoticed during the initial inspection. Finally, the participants had a positive impression about the usefulness of the HET. Conclusions: The HET is effective for identifying and classifying requirements errors and faults, thereby helping to improve the overall quality of the SRS and the software.
\end{abstract}

\section{Keywords}

Human Errors, Software Requirements, Software Quality Improvement

\section{INTRODUCTION}

The software development process is human-centric as developers interact with customers and users to document customer needs into software requirements and then transform those requirements into architecture, design, and ultimately

Permission to make digital or hard copies of all or part of this work for personal or classroom use is granted without fee provided that copies are not made or distributed for profit or commercial advantage and that copies bear this notice and the full citation on the first page.

Copyrights for components of this work owned by others than the author(s) must be honored. Abstracting with credit is permitted. To copy otherwise, or republish, to post on servers or to redistribute to lists, requires prior specific permission and/or a fee. Request permissions from Permissions@acm.org.

ESEM '16, September 08 - 09, 2016, Ciudad Real, Spain Copyright is held by the owner/author(s). Publication rights licensed to ACM.

ACM $978-1-4503-4427-2 / 16 / 09 \ldots \$ 15.00$

DOI: http://dx.doi.org/10.1145/2961111.2962596 code. Much research and experience has shown that developers introduce problems during various stages of the development process. Identifying and correcting such problems can be quite costly. Software engineering researchers have devoted significant attention to improving software quality. Most of this attention has traditionally focused on approaches that address the faults, i.e., the problems recorded in various software engineering artifacts. To provide a comprehensive picture of the problem, it is also important to understand and address the underlying cause of the faults, i.e. the human mental errors. Identifying these human mental errors can help developers understand why problems occurred, find and fix related faults, and prevent errors from happening in the future.

The development of the software requirements specification (SRS) is the first and most crucial stage in the software engineering lifecycle. During this phase, developers communicate with various stakeholders to elicit and document customer needs and specify the solution. Development of the SRS is especially defect-prone because of the ambiguities of natural language and the concomitant difficulty in communicating with all relevant stakeholders. If these SRS defects are not detected and repaired at the requirements stage, they become more expensive to fix. Developers often spend $40-50 \%$ of their effort fixing problems that should have been fixed earlier in the software lifecyce [1]. Therefore, our work focuses on identifying the human mental errors that caused the requirement defects to reduce the chances of those defects propagating to later stages.

Other researchers have recognized this need to understand the human errors that cause the faults, and have proposed various solutions. Root Cause Analysis helps developers identify systematic development problems, characterize the source of faults, and identify process improvement needs based on those sources [9]. Orthogonal Defect Classification provides in-process feedback to developers and helps them identify faults as early as possible [3]. Error Abstraction help developers identify the errors that underlie the faults detected during an inspection and provides guidance on how to reinspect to find additional errors and faults [7]. While all of these approaches seek to understand the source of problems, none of these have a strong connection to work in cognitive psychology, which has a long history of studying human errors and their manifestations and consequences in many domains.

Because the software development process is a humanbased activity, it is reasonable to investigate the applica- 
tion of insights from research in cognitive psychology. Requirements faults are often the result of failings in human thought. For example, a case study by Trevor and Jim found that software problems resulted from phenomena associated with human mental processes, which were not directly related to software engineering [14]. In another example, Lopes and Forster provided a proof of concept that the use of human error theories can improve the quality of software requirements [10]. These studies help establish that the use of human error theories can improve software quality by helping developers better understand problems in the development of the SRS and by reducing the number of inserted defects.

Employing error research from the cognitive psychology literature, we developed a taxonomy of human errors that can occur during the development of an SRS. Our Human Error Taxonomy (HET) was informed by a systematic literature review that combines research from software requirements with research from cognitive psychology. Working with a Cognitive Psychologist (the last author of this paper), we analyzed the human error literature to determine how cognitive theories can be applied to software engineering problems. As a result, our taxonomy is theoreticallygrounded in appropriate research results. Section 2.3 provides an overview of the HET.

The goal of this paper is to investigate the feasibility of using the HET to support the SRS inspection process. The primary contributions of this paper are (1) evaluation of the feasibility of the HET for supporting inspection of an SRS and (2) an analysis of the types of human errors that novice developers make while developing an SRS.

The remainder of this paper is organized as follows: Section 2 provides background. Section 3 provides a description of a feasibility study conducted to evaluate the effectiveness and efficiency of the proposed approach. Section 4 describes the analysis and result of the study. Section 5 summarizes and discusses the results. Section 6 discusses the threats to validity of this study, followed by a brief conclusion of this paper and ideas for future studies in Section 7 .

\section{BACKGROUND}

This section provides background information on topics relevant to our study. Section 2.1 describes previous research showing the value of building taxonomies of software requirements problems. Section 2.2 explains the process of error abstraction. Section 2.3 describes the HET that is the subject of this study.

\subsection{Fault-Based Taxonomies}

Because this work makes use of a taxonomy of problems, it is important to understand prior work on creating taxonomies of software problems. Researchers studying earlylifecycle quality improvement methods have developed taxonomies of software faults. Subsequent empirical evaluation showed that such taxonomies can help developers improve software quality $[4,5,11,2]$. However, these approaches cannot help developers identify the full spectrum of faults or how to improve development practices based on such faults [18]. These taxonomies are also incomplete [8]. Thus, there is a need for new approaches that extend existing taxonomies of problems, but overcome their shortcomings. Specifically, there is a need to focus on the sources of the problems (i.e. the human error).

\subsection{Error Abstraction Process}

The error abstraction process used in this paper is based on previous work by Lanubile et al. [7]. Error abstraction helps developers identify the root causes of requirements faults. Each inspector will be trained on how to abstract the underlying errors from related faults. The relationship between errors and faults is many-to-many, that is one error may cause multiple faults and one fault may be caused by multiple errors. For example, the error of lack of communication between end users and requirement engineers can lead to the faults of missing information or incorrect information. Thus, using the error abstraction will help inspectors understand the underlying causes for related faults and help them identify additional faults, related to the abstract error, in the SRS.

\subsection{Human Error Taxonomy (HET)}

Previously, Walia and Carver developed an initial Requirement Error Taxonomy (RET) to provide structure to the process of identifying human errors in the development of SRS documents [16]. They subsequently validated the usefulness of the RET through a series of empirical studies [17]. Key findings from these studies include (1) use of structured error information improves fault detection effectiveness compared with using only fault information and (2) the use of human error information is beneficial for the prevention of requirements problems. The primary weakness of the RET was that, while it was inspired by human error research in cognitive psychology, it lacked a strong and direct tie to existing human error taxonomies. To address this weakness, we developed the Human Error Taxonomy (HET).

In order to build a formal and theoretically sound error taxonomy, we conducted a systematic literature review. First, we analyzed how the findings from human error research can be applied to the software engineering domain. Then, we analyzed human error research published in the software engineering literature to determine the degree of fit to psychological literature on human errors. The results of this analysis was a set of 15 error classes that described common problems that occur during the development of SRS documents. The last step was to map these error classes into one of the existing human error taxonomies from cognitive psychology.

Then, we mapped information about requirement error from the software engineering literature to human error taxonomies from the cognitive literature. After examining a number of taxonomies, we chose the Slips, Lapses, and Mistakes taxonomy by Reason [13]. We chose this taxonomy because it establishes a strong association between the human information processing model and cognitive mechanisms like inattention, memory failures, and lack of knowledge. These cognitive mechanisms are easy to understand and relate to when classifying a given human error into the category of slip, lapse or mistake.

Slips and Lapses describe errors that occur when a planned action was not executed or completed as intended. A Slip error occurs when someone carries out a planned task incorrectly or in the wrong sequence. For example, someone intended to pour milk on his/her morning cereal but instead pours orange juice. Lapse errors are generally memory related failures. They occur when someone forgets a goal in the middle of a sequence of actions or omits a step in a 
routine sequence. For instance, someone enters a room to accomplish some task, but upon entering the room forgets the planned task. Conversely a Mistake is a planning error in that someone designed an incorrect plan to achieve the desired goal. In this error, the plan is executed properly, but the plan itself was inadequate to achieve the intended outcome. For example, suppose you are driving your usual route work and encounter traffic. You devise an alternate plan to travel to work via a different route. On your new route, you encounter unexpected road construction and must return to the original route. Your plan was wrong because your model of the city traffic pattern was wrong.

Full details of the HET and its development are beyond the scope of this paper. Table 1 provides an overview of the HET. The details can be found in a document on a webpage created for this paper: http://humanerrorinse.org/Studies/ 2015/Fall_UA/index.htm

One example of an error from the Environment class of the Mistake type in the HET and the resulting fault is:

Error: When documenting software requirements, the developers did not use a standard requirement specification template (e.g., the IEEE standard). In this case, the correct tools for requirement specification were not used.

Fault: Requirements about the system scope and performance were omitted.

\section{EXPERIMENT DESIGN}

The major goal of this study is to investigate the feasibility of using the HET to support the SRS inspection process. The following subsections describe the research questions and hypotheses, the participants, and study procedures.

\subsection{Research Questions and Hypotheses}

To address the overall study goal, we explored three distinct research questions. Along with each research question, we define specific research hypotheses.

RQ1: Does the HET provide a useful method of describing and classifying the errors and faults made during development of an SRS?

As described in Section 2.3, the HET is comprised of three high-level error types and fifteen detailed error classes. Based on our literature review, each of these error types and classes has both theoretical and empirical support. By incorporating previous reports of errors made during the development of an SRS, the error classes in the taxonomy are likely to re-occur at a later time in the same SRS development effort or in future SRS development efforts. The focus of this research question is to understand whether these error types and classes actually describe real problems that occur in the SRS. To ensure that the taxonomy is valid, we also need to understand whether all of the error types and classes are necessary.

Hypothesis 1: Developers will find errors and related faults that fit into each of the three high-level error types and the fifteen low-level error classes (i.e., the observed frequency will be greater than 0 ).

Based on our HET, the Mistakes error type has the largest number of error classes. The study of Kraemer and Carayon also pointed out that the mistake errors are the most common in the area of computer and information security [6].

Hypothesis 2: Developers will make more Mistake errors and faults.

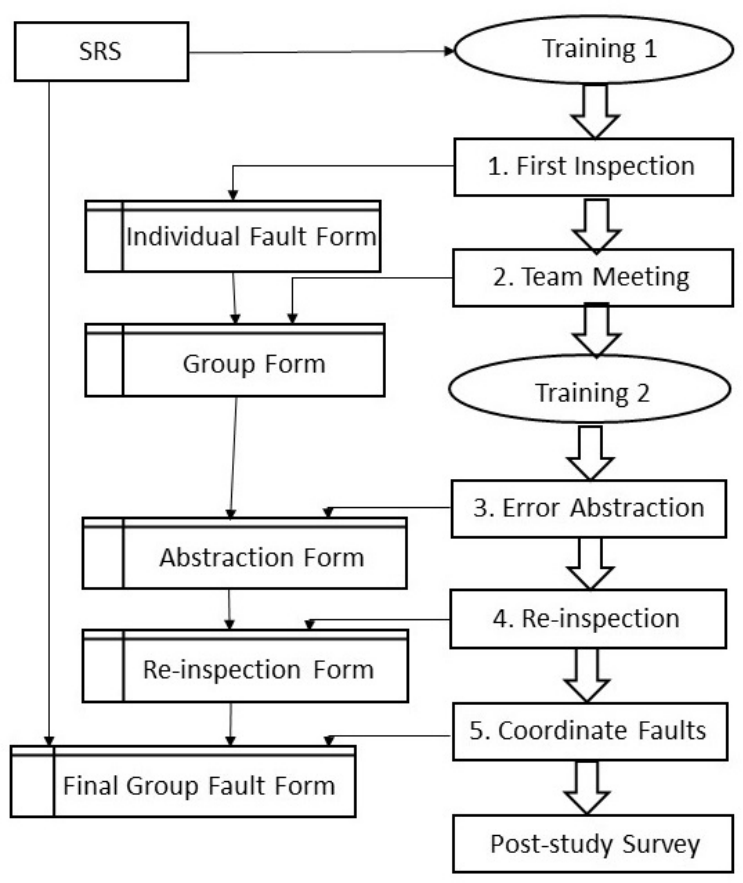

Figure 1: Experiment Procedure

RQ2: Do the errors identified by the HET lead to additional faults being identified during reinspection?

Once a development team becomes aware that they have made an error during the creation of the SRS, they may identify additional faults related to that error. To further understand the usefulness of the HET, we need to determine whether knowledge of errors can lead developers to find additional related faults.

Hypothesis 3: Development teams will find additional faults when reinspecting their SRS document guided by knowledge of existing errors (i.e., the observed frequency will be greater than 0$)$.

\section{RQ3: Do developers think the HET is helpful?}

This question focuses on the developers' subjective impression of the HET. Similar to the prior work of Walia and Carver [17], because error abstraction and classification is a subjective process, we also measured effectiveness through a developer survey after using the HET.

Hypothesis 4: Developers believe that the HET is useful for abstracting and classify requirement errors.

\subsection{Participants}

The study included twenty-eight senior-level undergraduate computer science students enrolled in the Fall 2015 capstone project course at The University of Alabama. The primary course goal was for student teams to proceed through requirements elicitation/documentation, design, implementation, and testing to build a complete software system. Separate from the design of this study, the course instructor (who is not part of the research team) divided the students into eight three- or four-person teams. Each team developed their own system (as described in Table 2) and all the requirements documents were all textual. 
Table 1: Human Error Taxonomy

\begin{tabular}{|c|c|c|}
\hline $\begin{array}{l}\text { Reason's } \\
\text { Taxonomy }\end{array}$ & Human Error Taxonomy & Error Description \\
\hline \multirow[t]{2}{*}{ Slips } & Clerical Errors (CE) & $\begin{array}{l}\text { Due to carelessness while performing mechanical transcrip- } \\
\text { tions from one format or from one medium to another. }\end{array}$ \\
\hline & $\begin{array}{l}\text { Lack of consistency in the requirement } \\
\text { specification (LC) }\end{array}$ & $\begin{array}{l}\text { occur when requirement authors are not able to articulate } \\
\text { and organize the requirements in a consistent manner, even } \\
\text { when they have a clear idea of user needs. }\end{array}$ \\
\hline \multirow[t]{2}{*}{ Lapses } & $\begin{array}{l}\text { Loss of information from stakeholders } \\
\text { (LI) }\end{array}$ & $\begin{array}{l}\text { Forgetting, discarding or failing to store information or doc- } \\
\text { uments provided by stakeholders. }\end{array}$ \\
\hline & $\begin{array}{l}\text { Accidentally overlooking requirements } \\
(\mathrm{AO})\end{array}$ & $\begin{array}{l}\text { Many requirements, both functional and non-functional, are } \\
\text { accidentally overlooked. }\end{array}$ \\
\hline \multirow{11}{*}{ Mistakes } & Application Errors (AE) & $\begin{array}{l}\text { Arise from a misunderstanding of the application or problem } \\
\text { domain. }\end{array}$ \\
\hline & Environment Errors (EE) & $\begin{array}{l}\text { Due to a misunderstanding or misuse of the hardware or } \\
\text { software environment of a given project. }\end{array}$ \\
\hline & Information Management Errors (IM) & Due to a lack of knowledge about standard procedures. \\
\hline & Wrong Assumptions (WA) & $\begin{array}{l}\text { Happens here is creation of mistaken assumptions about fea- } \\
\text { tures and opinions of stakeholders. }\end{array}$ \\
\hline & $\begin{array}{l}\text { Low Understanding of one another's } \\
\text { Roles (LU) }\end{array}$ & $\begin{array}{l}\text { Domain knowledge and perspectives vary between roles, } \\
\text { which necessitates considerable communication among mem- } \\
\text { bers of the software engineering team. }\end{array}$ \\
\hline & $\begin{array}{l}\text { Mistake belief that it is impossible to } \\
\text { sppcify non-functional requirements in } \\
\text { a verifiable form (MB) }\end{array}$ & $\begin{array}{l}\text { Major causes of this problem are the prevalent myths that it } \\
\text { is too costly, too difficult, and even impossible to produce } \\
\text { good requirements, especially nonfunctional requirements, } \\
\text { during the software engineering process. }\end{array}$ \\
\hline & $\begin{array}{l}\text { Not having a clear distinction between } \\
\text { client and users (CD) }\end{array}$ & $\begin{array}{l}\text { Fail to gather and analyze requirements from the userâÁ } \\
\text { point of view. }\end{array}$ \\
\hline & $\begin{array}{l}\text { Lack of awareness of sources of require- } \\
\text { ments (LA) }\end{array}$ & $\begin{array}{l}\text { Requirements gathering person is not aware of all stakehold- } \\
\text { ers which he/she should contact in order to gather the com- } \\
\text { plete set of user needs. }\end{array}$ \\
\hline & Problem-Solution Errors (PS) & $\begin{array}{l}\text { Due to not knowing, misunderstanding, or misuse of problem } \\
\text { solution processes. }\end{array}$ \\
\hline & Inadequate Requirements Process (IR) & $\begin{array}{l}\text { The requirements engineering plan is incomplete or inade- } \\
\text { quate for the current project. }\end{array}$ \\
\hline & Syntactic Errors (SE) & $\begin{array}{l}\text { Syntax describes the grammatical rules for specifying correct } \\
\text { arrangement of elements to produce meaning. }\end{array}$ \\
\hline
\end{tabular}

Table 2: Teams and System Description

\begin{tabular}{|c|c|c|c|}
\hline Team \# & System Name and Description & \# Page & \# Req \\
\hline 1 & Grade Keeper: manage student course information & 21 & 14 \\
\hline 2 & Tenant Tracker: provide communication between property managers and tenants & 22 & 12 \\
\hline 3 & Nutrition: keep track of the food the users have eaten & 15 & 7 \\
\hline 4 & Opus: share pictures of interesting items in the local community & 14 & 7 \\
\hline 5 & Robotics Score: manage the scores for a robotics competition & 15 & 19 \\
\hline 6 & Promo Pass: manage business promotion information for customers & 22 & 4 \\
\hline 7 & Bowling: create and manage a bowling game session & 17 & 13 \\
\hline 8 & Is Open: manage detailed information about local businesses & 8 & 9 \\
\hline
\end{tabular}

\subsection{Procedure}

The study procedure included two training sessions, five experimental steps, and a post-study survey. Figure 1 illustrates the process, which is described below.

Training 1 - Fault checklist technique: The goal of the initial training was to introduce the types of faults that can occur when developing an SRS. Using a requirements fault checklist [12], we provided detailed information about the important types of faults. We also trained the participants on how to use the checklist to inspect an SRS and on how to record the faults found during that inspection. This training session lasted approximately 15 minutes.

Step 1- First Inspection (Individual Inspection): We randomly assigned each participant to evaluate an SRS developed by another team. The participants then used the fault checklist (from Training 1) to inspect the assigned SRS and identify faults. Each participant completed an Individual Fault Form to list the faults found during the inspection. The output of this step was 28 Individual Fault Forms (one per participant).

Step 2 - Team Meeting to Consolidate Faults: Each team received the Individual Fault Forms submitted by the 
participants who inspected their SRS (Step 1). As a group, each team examined these fault lists to remove duplicates and consolidate the faults into one master list, which they recorded on the Group Form. The output of this step was eight Group Forms (one per team).

Training 2 - Error abstraction and classification: During the first 20 minutes, we trained the participants on the error abstraction process, including how to abstract errors from faults. Then we spent 20 minutes training the participants on the HET and how to use it to abstract and classify requirements errors. We described each HET error class and provided an illustrative detailed example.

Step 3 - Error Abstraction: Based on Training 2, each team used the HET to abstract and classify the errors recored on their Group Form from Step 2. They recorded these errors in an Abstraction Form. The output of this step was eight Abstraction Forms (one per team).

Step 4 - Re-inspecting the SRS: Using the errors abstracted in Step 3, each participant individually reinspected their own SRS document to identify any additional faults related to these errors (i.e. faults not found by their classmates in Step 1). The participants recorded these additional faults on the Reinspection Form. The output of this step was 28 Reinspection Forms (one per participant).

Step 5 - Coordinating the individual fault lists: This step is equivalent to Step 2, except that the teams used the faults found in the reinspection (Step 4). Each team produced a Final Group Fault Form reporting the agreedupon list of faults. The output of this step was eight Final Group Fault Forms (one per team).

Post-study Survey: At the end of the study, each participant provided subjective feedback about the error abstraction process and the use of the HET. The details of the survey and the specific questions are listed in Table 6 in Section 4.3.

The study materials, including training slides and information about the HET can be found here:

http://humanerrorinse.org/Studies/2015/Fall_UA/index.htm.

\subsection{Data Cleaning and Analysis Process}

After completing the study procedure, we determined that Teams 7 and 8 did not follow steps 2-5 correctly and failed to provide the required data. The members of these teams did perform Step 1 correctly to provide input to the other teams. Therefore, for the remainder of the paper, we report the results for the six teams that followed the instructions for steps 2-5. These six teams contained a total of twentyone participants. For the statistical analysis, selected an alpha value of 0.05 to judge the statistical significance of the results.

\section{ANALYSIS AND RESULTS}

This section provides a detailed analysis of the data collected during this study. The analysis is organized around the hypotheses posed in Section 3.1.

\subsection{RQ1: Does the HET provide a useful method of describing and classifying the errors and faults made during development of an SRS?}

Because we are interested in characterizing the overall usefulness of the HET, for the analysis in this section, we include data from the entire process (i.e. the Individual

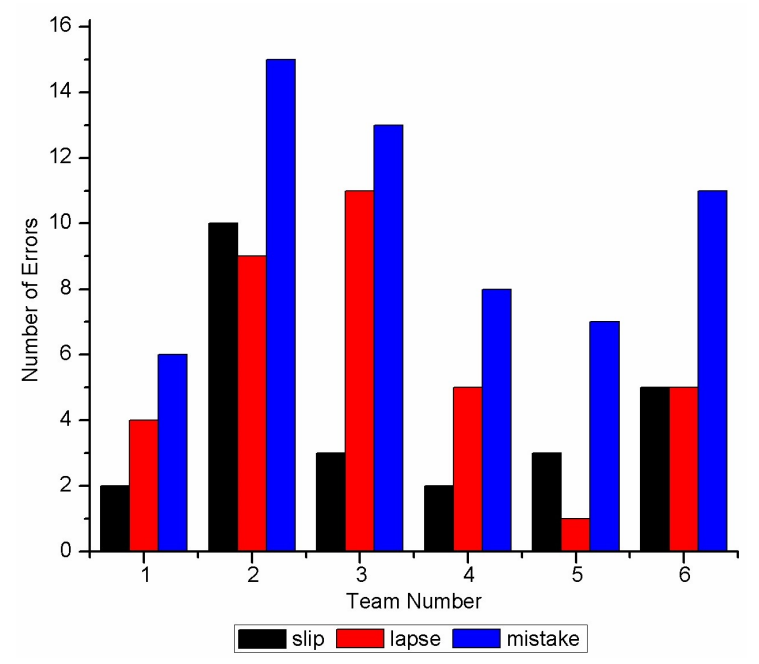

Figure 2: Team Errors by Error Type

Fault Forms, Group Forms, Abstraction Forms, Reinspection Forms, and Final Group Fault Forms).

H1: Developers will find errors and related faults that fit into each of the three high-level error types and the fifteen low-level error classes (i.e., the observed frequency will be greater than 0 ).

First, for the high-level error types, Figure 2 shows that all six teams made errors from each of the three error types. We ran three one-sample t-tests to determine whether the frequency errors of each type was significantly greater than 0 . In all three cases, the number of errors was significantly higher than 0: Slips $(\mathrm{p}=.01)$, Lapses $(\mathrm{p}=.005)$, and Mistakes $(\mathrm{p}=.001)$.

Figure 3 shows the distribution of the faults (grouped by error type) made by each team. Similar to the data on errors, this data shows that all six teams also made faults that are classified into each of the three error types. We again ran one-sample t-tests to see if the frequency of these faults is significantly greater than 0 . Again, in all three cases the number of faults was significantly greater than 0: Slips $(p=.021)$, Lapses $(p=.006)$, and Mistakes $(p=.002)$.

Second, Table 3 shows the distribution of the errors among the 15 low-level error classes for each team. According to the table, eleven of the fifteen error classes were represented in at least one SRS document. Similar to the analysis of the error types, we conducted a series of one-sample t-tests to determine if the number of faults in each class was significantly greater than 0 across all teams. The shaded cells in the last column of Table 3 indicate which error classes had an error frequency that was significantly higher than 0 .

Table 4 shows the distribution of the faults (grouped by error class) for each team. Similar to the errors, this data shows that developers also make faults that are classified into most, but not all, of the error classes. Not surprisingly, the same error classes that were missing errors are also missing faults. The shaded cells in Table 4 indicate the error classes for which the frequency of errors was significantly greater than 0 .

H2: Developers will make more Mistake errors and faults.

Table 5 shows the percentage of errors and faults from 


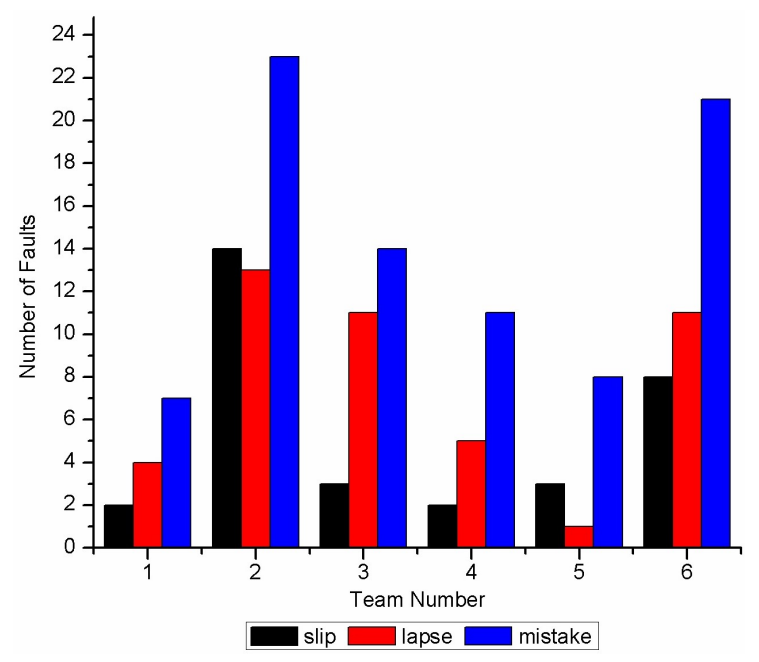

Figure 3: Team Faults by Error Type

Table 3: Team Errors by Error Class

\begin{tabular}{|c|c|c|c|c|c|c|c|}
\hline $\begin{array}{c}\text { Error } \\
\text { types }\end{array}$ & T1 & T2 & T3 & T4 & T5 & T6 & $\begin{array}{c}\% \text { of total } \\
\text { errors }\end{array}$ \\
\hline CE & - & 2 & 3 & 1 & 3 & - & $8 \%$ \\
\hline LC & 2 & 8 & - & 1 & - & 5 & $13 \%$ \\
\hline LI & - & 4 & 3 & - & - & 3 & $8 \%$ \\
\hline AO & 4 & 5 & 8 & 5 & 1 & 2 & $21 \%$ \\
\hline AE & - & - & 1 & - & 1 & - & $2 \%$ \\
\hline EE & 2 & - & - & - & 1 & - & $3 \%$ \\
\hline IM & 2 & 6 & - & 7 & 3 & - & $15 \%$ \\
\hline WA & 2 & 3 & - & - & - & 6 & $9 \%$ \\
\hline LU & - & - & - & - & - & - & - \\
\hline MB & - & - & 10 & 1 & 1 & - & $10 \%$ \\
\hline CD & - & - & - & - & - & - & - \\
\hline LA & - & - & - & - & - & - & - \\
\hline PS & - & - & - & - & - & - & - \\
\hline IR & - & - & 1 & - & 1 & 5 & $6 \%$ \\
\hline SE & - & 6 & 1 & - & - & - & $6 \%$ \\
\hline
\end{tabular}

shaded cells indicate result was significantly greater than 0

Table 4: Team Faults by Error Class

\begin{tabular}{|c|c|c|c|c|c|c|c|}
\hline $\begin{array}{c}\text { Error } \\
\text { types }\end{array}$ & T1 & T2 & T3 & T4 & T5 & T6 & $\begin{array}{c}\% \text { of total } \\
\text { faults }\end{array}$ \\
\hline CE & - & 2 & 3 & 1 & 3 & - & $6 \%$ \\
\hline LC & 2 & 12 & - & 1 & - & 8 & $14 \%$ \\
\hline LI & - & 8 & 3 & - & - & 8 & $12 \%$ \\
\hline AO & 4 & 5 & 8 & 5 & 1 & 3 & $16 \%$ \\
\hline AE & - & - & 1 & - & 1 & - & $1 \%$ \\
\hline EE & 2 & - & - & - & 1 & - & $2 \%$ \\
\hline IM & 3 & 7 & - & 10 & 3 & - & $14 \%$ \\
\hline WA & 2 & 9 & - & - & - & 9 & $12 \%$ \\
\hline LU & - & - & - & - & - & - & - \\
\hline MB & - & - & 10 & 1 & 1 & - & $7 \%$ \\
\hline CD & - & - & - & - & - & - & - \\
\hline LA & - & - & - & - & - & - & - \\
\hline PS & - & - & - & - & - & - & - \\
\hline IR & - & - & 1 & - & 2 & 12 & $9 \%$ \\
\hline SE & - & 7 & 2 & - & - & - & $6 \%$ \\
\hline
\end{tabular}

shaded cells indicate result was significantly greater than 0
Table 5: Comparison of Error Types

\begin{tabular}{|c|c|c|c|c|}
\hline Variable & Slip & lapse & mistakes & p-value \\
\hline Total Errors & $21 \%$ & $29 \%$ & $50 \%$ & $<0.001$ \\
\hline Total Faults & $20 \%$ & $28 \%$ & $52 \%$ & $<0.001$ \\
\hline
\end{tabular}

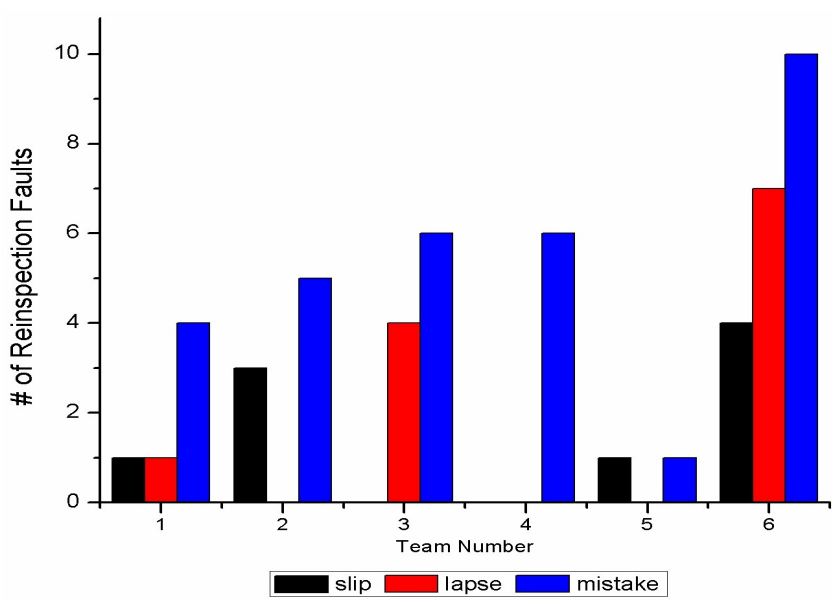

Figure 4: Faults found during Reinspection Grouped by Error Type

each error class across all teams. The results of the $\chi^{2}$ test (against a uniform distribution) shows that the distribution was significantly different from uniform. Examining the distribution shows that Mistake errors were responsible for half of the errors and faults.

\subsection{RQ2: Do the errors identified by the HET lead to additional faults being found dur- ing reinspection?}

This question focuses only on the value of the errors during the reinspection process. So, for this question we analyze on the data reported on the Reinspection Forms to test this hypothesis.

H3: Development teams will find additional faults when reinspecting their SRS document guided by knowledge of existing errors (i.e., the observed frequency will be greater than 0).

During the Reinspection Step (Step 4 in Figure 1), members of each team inspected their own SRS guided by the errors identified in the Error Abstraction Step (Step 3 in Figure 1). Because the reinspection was guided by the already-identified errors, the teams were searching only for new faults (not new errors). Figure 4 shows the number of faults found during the reinspection by error type. The results show, similar to RQ1, faults related to the Mistake error type were the most common in the reinpsection. The results of the one-sample t-test show that the frequency of faults found in the Slip and Mistake types were significantly greater than 0 ( $\mathrm{p}=.038$ and .007 respectively).

Figure 5 shows the number of faults found during the reinspection by error class. The one-sample t-tests did not show that the frequencies of any individual error class was significantly greater than 0 . 
Table 6: Post-Study Survey

\begin{tabular}{|c|c|c|}
\hline Characteristic & Item & Statement \\
\hline \multirow{6}{*}{ Usefulness } & Usefulness_1 & $\begin{array}{l}\text { The HET is helpful for } \\
\text { identifying faults }\end{array}$ \\
\hline & Usefulness_2 & The HET is complete \\
\hline & Usefulness_3 & $\begin{array}{l}\text { The HET will be useful } \\
\text { on future project }\end{array}$ \\
\hline & Usefulness_4 & $\begin{array}{l}\text { The HET is helpful in } \\
\text { improving the SRS }\end{array}$ \\
\hline & Usefulness_5 & $\begin{array}{l}\text { The HET is helpful to } \\
\text { detect overlooked faults }\end{array}$ \\
\hline & Usefulness_6 & $\begin{array}{l}\text { The effort on using } \\
\text { HET is valuable }\end{array}$ \\
\hline \multirow[t]{2}{*}{ Intuitiveness } & Intuitiveness_1 & The HET is intuitive \\
\hline & Intuitiveness_2 & $\begin{array}{l}\text { I am confident that } \\
\text { errors represent real } \\
\text { problem }\end{array}$ \\
\hline $\begin{array}{l}\text { Confidence } \\
\text { (con) }\end{array}$ & Confidence & $\begin{array}{l}\text { I am confident in the } \\
\text { error abstraction }\end{array}$ \\
\hline $\begin{array}{l}\text { Understand } \\
\text { (under) }\end{array}$ & Understand & $\begin{array}{l}\text { The HET is easy } \\
\text { to understand }\end{array}$ \\
\hline $\begin{array}{l}\text { Classification } \\
\text { (cla) }\end{array}$ & Classify & $\begin{array}{l}\text { The HET is easy to use } \\
\text { to classify errors }\end{array}$ \\
\hline $\begin{array}{l}\text { Abstraction } \\
\text { (abs) }\end{array}$ & Abstract & $\begin{array}{l}\text { The HET is easy to use } \\
\text { to abstract errors }\end{array}$ \\
\hline $\begin{array}{l}\text { Helpful } \\
\text { (help) }\end{array}$ & Helpful & $\begin{array}{l}\text { The HET is helpful for } \\
\text { understanding the faults }\end{array}$ \\
\hline $\begin{array}{l}\text { Orthogonality } \\
\text { (ort) }\end{array}$ & Orthogonal & The HET is orthogonal \\
\hline $\begin{array}{l}\text { Clear training } \\
\text { (clear) }\end{array}$ & training & $\begin{array}{l}\text { The training I received } \\
\text { on HET is clear }\end{array}$ \\
\hline
\end{tabular}

\subsection{RQ3: Do developers think the HET is help- ful?}

Using the data from the post-study survey, we tested the following hypothesis.

H4: Developers believe that the HET is useful for abstracting and classifying requirement errors.

Based on previous studies by Walia and Carver to obtain subjective feedback on the usefulness of the RET [15], we developed a post-study survey to evaluate nine specific characteristics of the HET: usefulness, intuitiveness, confidence, understandable, classification, abstraction, helpful, orthogonality and clear-training materials. Because we wanted to consider usefulness and intuitiveness a little more broadly than in the previous work, we added some dimensions to those two attributes. The participants were asked to use a 5-point scale ranging from 1 - strongly disagree to 5 strongly agree to indicate their level of agreement about a series of statements related to these characteristics. Table 6 lists the statements included on the survey. As the survey was not mandatory, only 12 students responded.

Figures 6,7 , and 8 shows the distribution of the responses for each characteristic (Figure 6 has the characteristics with only one question each and Figures 7 and 8 have the characteristics with multiple questions).

The results show that, in general, the participants viewed the HET positively for all attributes. There are no cases

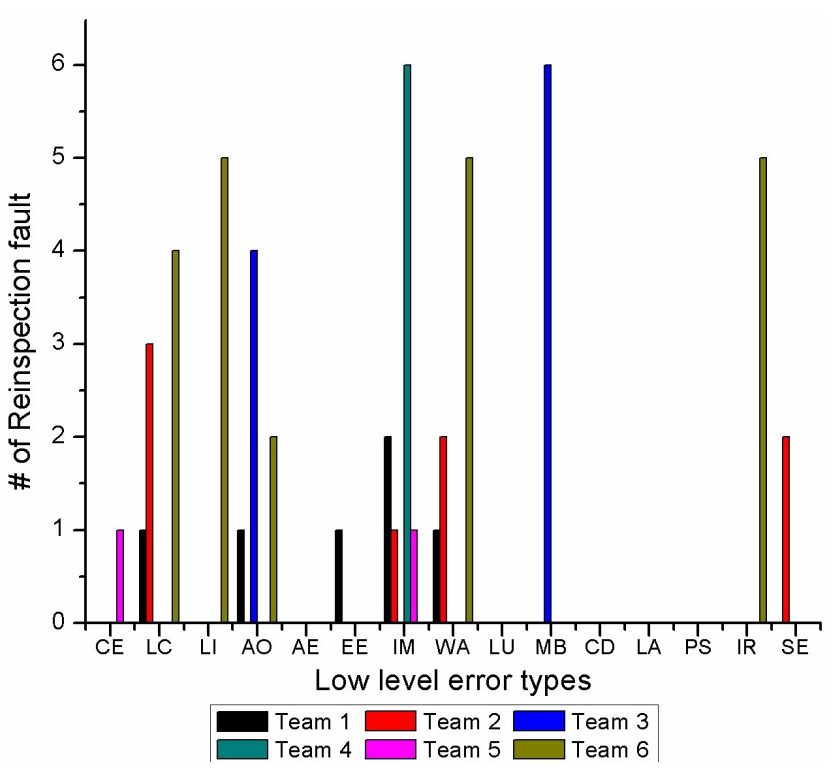

Figure 5: Faults found during Reinspection Grouped by Error Class

for which an attribute received more low ratings than high ratings. To determine whether these attributes were rated significantly higher than the mid-point of the scale (3), we conducted a one-sample t-test for each characteristics. The results in Table 7 show that respondents viewed the HET significantly positive on most, but not all, attributes.

\section{SUMMARY AND DISCUSSION OF THE RESULTS}

The major focus of this study was to investigate the feasibility of using the HET to guide the inspection of SRS documents. If we can establish the feasibility of the HET, then future studies can provide more detailed evaluations of various aspects of the HET to ensure its usefulness in real development environments. To provide insight into that goal, this section provides answers to the three research questions posed in Section 3.1 with a detailed discussion and interpretation of the results in Section 4.

\subsection{RQ1: Does the HET provide a good method of describing and classifying the errors and faults made while developing the SRS?}

This section examines the results in terms of the error types and the error classes. Then it describes some additional analysis to provide more insight into the results.

\subsubsection{High-level Error Types}

The results from Section 4.1 showed that the frequency of errors and faults committed is significantly higher than 0 for each of the error types. This result suggests that all three error types in the HET are important because developers made errors and faults in each one of them. The results also showed that the most common type of error and fault was Mistake. There are three potential explanations for the higher number of Mistakes. First, in the HET, there are more error classes in the Mistake error type than in the other two error types. Second, Mistakes usually occur because of 


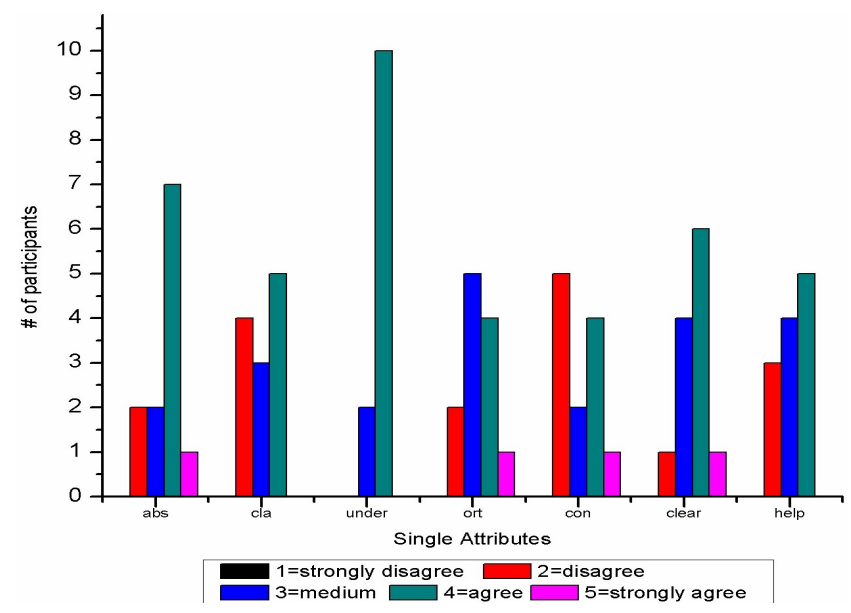

Figure 6: Responses for Single Attribute Characteristics

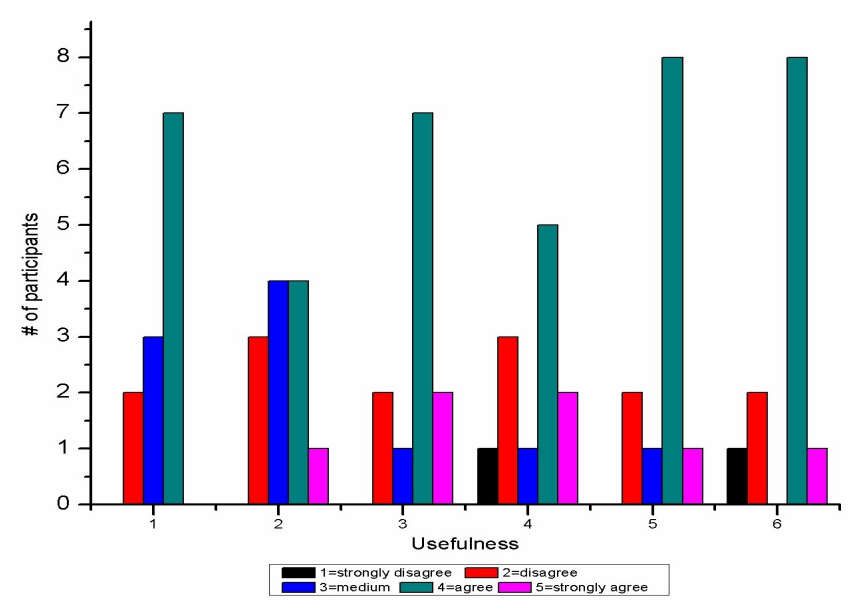

Figure 7: Responses for Usefulness Characteristic

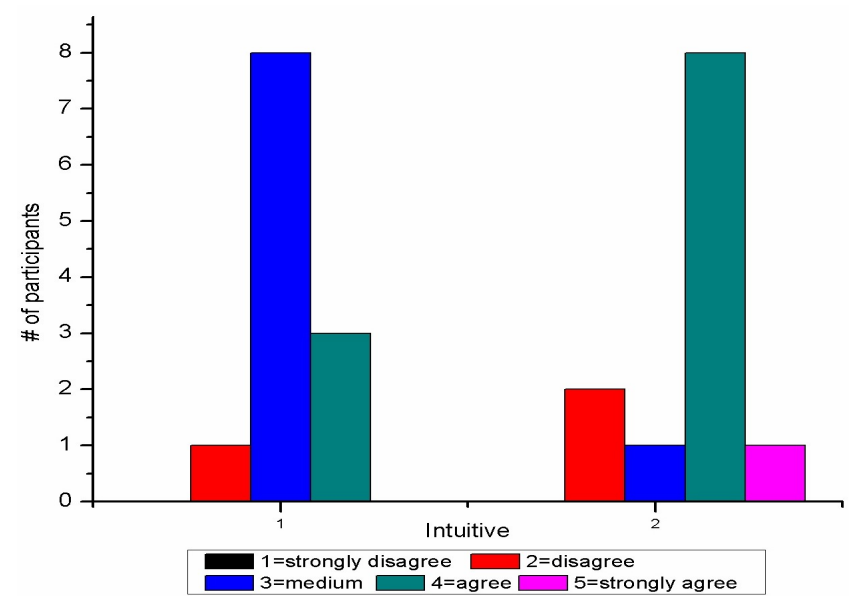

Figure 8: Responses for Intuitiveness Characteristic
Table 7: One sample T-test of nine characteristics

\begin{tabular}{|c|c|c|c|}
\hline Error types & p-Value & $\begin{array}{c}\text { Lower } \\
\text { Interval }\end{array}$ & $\begin{array}{c}\text { Upper } \\
\text { Interval }\end{array}$ \\
\hline abs & 0.023 & 0.01 & 1.16 \\
\hline clas & 0.377 & -.49 & 0.66 \\
\hline under & 0.00 & 0.59 & 1.08 \\
\hline ort & 0.110 & -.23 & .90 \\
\hline con & 0.398 & -.61 & 0.77 \\
\hline clear & 0.014 & 0.08 & 1.09 \\
\hline help & 0.252 & -.36 & 0.70 \\
\hline usefulness_1 & 0.013 & 0.10 & 1.45 \\
\hline usefulness_2 & 0.021 & 0.01 & 1.78 \\
\hline usefulness_3 & 0.011 & 0.14 & 1.36 \\
\hline usefulness_4 & 0.197 & -.49 & 1.16 \\
\hline usefulness_5 & 0.013 & 0.10 & 1.23 \\
\hline usefulness_6 & 0.012 & 0.10 & 1.27 \\
\hline intuitiveness_1 & 0.083 & -.20 & 0.53 \\
\hline intuitiveness_2 & 0.013 & 0.10 & 1.23 \\
\hline shaded cells indicate result was significantly greater than 3
\end{tabular}

a lack of knowledge, while Slips and Lapses tend to occur in familiar situations when attention is diverted. Finally, slips and lapses are often recognized and corrected quickly, and so may not make their way into the SRS documents, while mistakes tend to be more difficult to recognize and therefore persist longer. In this study, the participants were university students who lack experience and knowledge about software engineering in general and software requirements specifically. Therefore, they may be more prone to Mistakes.

\subsubsection{Low-level Error Classes}

The results from Section 4.1 also showed that developers made errors and faults in most of the fifteen low level error types. The most common error types are lack of consistency in the requirement specification, loss of information from stakeholders, accidentally overlooking requirements, information management errors, and wrong assumptions. Conversely, four error types Low understanding of one another's roles, Not having a clear distinction between client and users, Lack of awareness of sources of requirements, and Problemsolution errors were not made by the developers in this study (at least there were no errors of these types identified during the inspections). From this one study, we are not yet ready to remove these error types. It is possible that these were not relevant to the SRS documents developed in this study. It is also possible that developers made errors of these types, but just did not detect them during the inspection process. We still need to conduct additional studies before making any final conclusions about the contents of the HET.

Further analysis of these results reveals that different teams made different errors and resulting faults. No single error class was responsible for the majority of the errors or faults. For example, Teams 2 and 4 made a large number of Information Management errors and faults, while the majority of Team 3's errors and faults resulted from the Mistaken belief that it is impossible to specify non-functional requirements in a verifiable form and Team 6 made the majority of its errors and faults because of an Inadequate Requirements Process.

Another interesting observation is that some error classes are more common across all teams than others. For example, all teams made an error and related fault because of Acci- 
dentally Overlooking Requirements. Four of the six teams made errors and faults related to Clearical Errors, Lack of Consistency in the Requirements Specification, and Information Management. Other error types were not present at all or were only present on two or three teams. These observations suggest that the SRS development process varies across teams leading different teams to make different errors and faults.

These team differences could arise through many different reasons. Individual developers may have specific strengths and weaknesses in software development, team dynamics may play a role, and the characteristics of the system under design may place distinct strains on the development effort. Because our teams all worked on different projects with different members, we cannot analyze the contributing roles of such factors. These factors are candidates for exploration in future studies.

\subsubsection{Additional Analyses}

Our analysis of the results showed that three teams (T1, T3, and T6) did not classify all abstracted errors into the HET. To complete the dataset and ensure that no information was omitted, the first author classified those errors into their appropriate error classes and types. We report these results here as a separate analysis rather than as part of the main results since we did the classification rather than the participants. This step resulted in 18 additional errors (9 in Team 1, 2 in Team 3, and 7 in Team 6) and 12 corresponding faults (10 in Team 1, 2 in Team 3, and 9 in Team $6)$. This subjective classification did not change the overall distribution of errors or faults.

\subsection{RQ2: Do the errors identified by the HET lead to additional faults being indetified during reinspection?}

One of the goals of this feasibility study was to understand whether knowledge of errors would aid developers in finding additional faults related to those errors. The main idea is that once a team becomes aware that they have committed an error (that is some human mental failing) in the SRS development, it is likely that additional faults related to that error are also present in the SRS. The amount of time that development teams can devote to inspections may be limited. The results from Section 4.2 showed that all teams found additional faults during the reinspection. If we can show that knowledge of errors can lead to identification of additional related faults, then teams might be able to better focus their effort during a reinspection task.

One of the limitations of the feasibility study is the lack of a control group. The goal of this study was not to compare HET to another approach. As a result, we cannot draw a conclusion about whether development teams would have found more or less faults during a reinspection without guidance from the error information. The goal of this study was to show that use of error information could lead to detection of additional faults. The next step in our work is to conduct control group studies to determine whether the insights provided by the HET provide more assistance to a developer than other approaches (e.g. a simple reinspection using the same fault checklist as the first inspection).

\subsection{RQ3: Do developers think the HET is help- ful?}

In order for an approach like the HET to be adopted, it must show objective value (RQ1 and RQ2) and subjective value. That is, if developers do not think the approach is helpful or do not like the approach they are less likely to use it, regardless of whether the objective data shows it to be effective. The results from Section 4.3 showed that overall the participants had a favorable view of the HET. Most participants either agreed or strongly agreed with each characteristic. The participants indicated that the HET was helpful for abstracting and classifying faults into errors and that it was helpful for identifying additional faults during the reinspection. We did not gather any qualitative feedback through interviews or additional survey questions. In our future studies, we will gather more detailed feedback to allow us to improve the HET.

\section{THREATS TO VALIDITY}

Because this study was a feasibility study with the primary goal of determining whether there was enough evidence to proceed with more thorough evaluations, it has a number of threats to validity. In our future studies, we will address these validity threats through more complex designs.

\section{Internal Validity}

There are two primary internal validity threats: mortality and lack of a control group. Regarding mortality, only six of the eight teams submitted valid data. Even though it is possible that the results would change if we had data from all eight teams, we have no evidence to suggest that the two missing teams would have behaved any differently. Furthermore, only 12 participants responded to the poststudy survey. It is possible that only those participants who viewed the HET as helpful took time to respond to the suvey. But again, we have no evidence that those who responded are different from those that did not.

The lack of a control group threat manifested itself in two ways. First, because we did not have developers use other approaches for classifying errors (like the RET) we cannot conclusively determine that the HET is the most effective approach. Second, because we did not have a control group perform a reinspection using some other approach, like a second fault-based inspection, we cannot be sure how many of the additional faults found during the reinspection were truly due to the use of the HET. We will eliminate this threat by adding a control group in the future study.

\section{External Validity}

The participants were building a realistic system to solve a problem they encountered in their own lives. However, the participants were still undergraduate students in a classroom environment, rather than professional developers in a real environment.

\section{Construct Validity}

Because the SRS documents were developed as part of the study, we do not know the real number of faults and errors present. Therefore, our conclusions are based only on the errors and faults actually detected. 


\section{CONCLUSIONS AND FUTURE WORK}

The results of this feasibility study showed a positive impact of the HET both objectively and subjectively. Objectively, developers were able to abstract errors from faults and classify those errors into the HET and were able to use those classified errors to find additional faults during a reinspection. Subjectively, the participants rated the HET positively on a number of key characteristics.

Regarding the HET itself, the study showed that the three high-level error types all made a useful contribution to detection and classification of errors and faults. Likely because of the low experience of the participants, Mistakes were the most common error type. We anticipate that if we conducted follow-on studies with participants of different levels of experience, we may see slightly different distributions of errors and faults.

Additionally, all but four of the low-level error classes were present in the SRSs from the teams in this study. These results indicate that overall the HET contains the right types of errors to describe the problems that occur during SRS development.

The study in this paper is a feasibility study geared towards establishing that there is enough evidence to proceed with more formal evaluation of the HET. To address some of the limitations of the feasibility study, we plan to conduct additional controlled experiments. We will run this study in the same capstone course. We will give different teams different error abstraction approaches and compare their performance in detecting faults and errors in an SRS. We will also study the usefulness of the HET for defection and prevention by teaching students about it prior to the development of their SRS to understand whether understanding the types of errors that they can make will prevent developeres from making those errors. Ultimately, we plan to replicate these studies with professional developers to validate the results in realistic situations.

\section{ACKNOWLEDGMENTS}

This work was supported by National Science Foundation Awards 1421006 and 1423279 . We thank the students who participated in the study. We also thank Dr. Jeff Gray for allowing us to conduct the study in his course.

\section{REFERENCES}

[1] B. Boehm and V. R. Basili. Software defect reduction top 10 list. Foundations of empirical software engineering: the legacy of Victor R. Basili, 426, 2005.

[2] J. Carver. The impact of background and experience on software inspections. Empirical Software Engineering, 9(3):259-262, 2004.

[3] R. Chillarege, I. S. Bhandari, J. K. Chaar, M. J. Halliday, D. S. Moebus, B. K. Ray, and M.-Y. Wong. Orthogonal defect classification-a concept for in-process measurements. Software Engineering, IEEE Transactions on, 18(11):943-956, 1992.

[4] B. Freimut, C. Denger, and M. Ketterer. An industrial case study of implementing and validating defect classification for process improvement and quality management. In Software Metrics, 2005. 11th IEEE International Symposium, pages 9-19. IEEE, 2005.

[5] J. H. Hayes. Building a requirement fault taxonomy: Experiences from a nasa verification and validation research project. In Software Reliability Engineering, 2003. ISSRE 2003. 14th International Symposium on, pages 49-59. IEEE, 2003.

[6] S. Kraemer and P. Carayon. Human errors and violations in computer and information security: The viewpoint of network administrators and security specialists. Applied ergonomics, 38(2):143-154, 2007.

[7] F. Lanubile, F. Shull, and V. R. Basili. Experimenting with error abstraction in requirements documents. In Software Metrics Symposium, 1998. Metrics 1998. Proceedings. Fifth International, pages 114-121. IEEE, 1998.

[8] C. Lawrence and I. Kosuke. Design error classification and knowledge management. Journal of Knowledge Management Practice, 10(9):72-81, 2004.

[9] M. Leszak, D. E. Perry, and D. Stoll. A case study in root cause defect analysis. In Proceedings of the 22nd international conference on Software engineering, pages 428-437. ACM, 2000.

[10] M. E. R. F. Lopes and C. H. Q. Forster. Application of human error theories for the process improvement of requirements engineering. Information Sciences, 250:142-161, 2013.

[11] C. Masuck. Incorporating a fault categorization and analysis process in the software build cycle. Journal of Computing Sciences in Colleges, 20(5):239-248, 2005.

[12] A. A. Porter, L. G. Votta Jr, and V. R. Basili. Comparing detection methods for software requirements inspections: A replicated experiment. Software Engineering, IEEE Transactions on, 21(6):563-575, 1995.

[13] J. Reason. Human error. Cambridge university press, 1990.

[14] C. Trevor, S. Jim, C. Judith, and K. Brain. Human error in the software generation process. University of Technology, Loughborough, England, 1994.

[15] G. S. Walia, J. Carver, and T. Philip. Requirement error abstraction and classification: an empirical study. In Proceedings of the 2006 ACM/IEEE international symposium on Empirical software engineering, pages 336-345. ACM, 2006.

[16] G. S. Walia and J. C. Carver. A systematic literature review to identify and classify software requirement errors. Information and Software Technology, 51(7):1087-1109, 2009.

[17] G. S. Walia and J. C. Carver. Using error abstraction and classification to improve requirement quality: conclusions from a family of four empirical studies. Empirical Software Engineering, 18(4):625-658, 2013.

[18] J. C. Westland. The cost of errors in software development: evidence from industry. Journal of Systems and Software, 62(1):1-9, 2002. 